\title{
Fibrome cémento-ossifiant du maxillaire : difficultés pour le diagnostic différentiel et la classification
}

\author{
Cementoossifying fibroma of the upper jaw: \\ problems in typing and differential diagnosis
}

BRUNO COURTOIS, CARLOS MADRID, DAMIEN DURAN, MARIE-PIERRE LABADIE

\begin{abstract}
RÉSUMÉ
Le fibrome cémento-ossifiant est une tumeur fibro-osseuse des maxillaires qui trouve son origine dans une anomalie de développement du ligament desmodontal. II se caractérise par un mode d'évolution lent et asymptomatique. La lésion prédomine chez la femme, avec une localisation mandibulaire préférentielle dans la région prémolo-molaire. Le traitement, exclusivement chirurgical, consiste à cliver la masse calcifiée dont la surface est lisse. La récidive est rare après exérèse. Cette observation concerne une femme de 43 ans, qui présente une tuméfaction ligneuse de la cavité buccale, intéressant le maxillaire droit, de la tubérosité à la région centro-palatine. La formation est sessile et semble faire corps avec l'os. On retrouve au pôle caudal de la lésion une ulcération en regard de la molaire mandibulaire antagoniste, le reste de la muqueuse est d'aspect normal. Le bilan radiologique révèle une image ostéolytique, circonscrite par un liseré d'ostéocondensation bien marqué et parsemée de fines radio-opacités ayant une disposition radiaire à la TDM. La lésion est appendue à la crête maxillaire édentée, à l'exception d'un reste radiculaire de 18. Le diagnostic de fibrome cémentoossifiant a été retenu. L'examen histopathologique de la pièce opératoire a confirmé ce diagnostic. Au stade initial, l'aspect histopathologique du fibrome cémento-ossifiant n'est guère différent de celui de la dysplasie ostéo-cémentaire péri-apicale et de celui de la dysplasie fibreuse des os, ce qui complique le diagnostic différentiel au stade initial. Son diagnostic repose donc sur des critères épidémiologiques, cliniques et surtout radiologiques concordants. La place du fibrome cémento-ossifiant dans la classification des tumeurs odontogéniques est à repenser dans le cadre d'une refonte de la classification des lésions fibro-osseuses dites à point de départ desmodontal. (Med Buccale Chir Buccale 2004; 10: 21-30)
\end{abstract}

mots clés : fibrome cémento-ossifiant, tumeur fibro-osseuse, diagnostic radiologique, diagnostic histopathologique.

\section{SUMMARY}

The cementoossifying fibroma is a benign neoplastic fibroosseous lesion from periodontal ligament origin. It grows slowly and is frequently asymptomatic. The tumour can affect patients over a wide age range, but most commonly appears between 20 and 40 years of age. There is a female preference and the lesion occurs mainly in the mandibule and is primarily found in the premolar and molar region. Management is exclusively surgical and allows the excision of an easily cleavable smooth-surfaced calcified mass. This case report is about a 43 year old female presenting a bony hard swelling of the right maxillary from the tuberosity to the midpalatine line. The neoplasm is intimately fixed to the underlying bone by a large foot. The overlying mucosa is normal except one ulcer on the top of 
the lesion caused by a mandibular molar. Radiologic examination shows an osteolytic, well-described radiolucency with, on CT slices, fine radiating radiopaque foci. The lesion originates from the upper jaw crest which is edentulous, except one root of 18. Surgical excision was performed under general anaesthesia. The microscopic examination shows an outlying fibroblastic proliferation surrounding orthoblastic stuctures with dystrophic calcifications and in some places a pattern of fibrous connective tissue matched with steady lamellar trabeculae. The conclusion of the pathologist confirms the clinical diagnosis of cementoossifying fibroma. Microscopic features of the cementoossifying fibroma make difficult to distinguish it, in early stages, from other fibroosseous lesions such like periapical cementoosseous dysplasia and fibrous dysplasia. Therefore, its diagnosis is mainly based on epidemiologic, clinical and radiologic corroborating data. No practical distinction can be made between so-called cementifying and ossifying fibroma and they are now categorized as cementoossifying fibroma while the term "ossifying fibroma" must be kept to extragnathic agressive or juvenile forms. The position of the cementoossifying fibroma within the histological classification of odontogenic tumours should take a fresh look according to a rewriting of the classification of the fibroosseous lesions from periodontal ligament origin. (Med Buccale Chir Buccale 2004; 10: 21-30)

key words : cementoossifying fibroma, fibroosseous tumour, microscopic diagnosis, radiologic diagnosis.

médecine

buccale

chirurgie

buccale

VOL. $10, \mathrm{~N}^{\circ} 1$ 2004

page 22
Dans la classification des tumeurs odontogéniques de l'OMS, le fibrome cémento-ossifiant a été placé dans le groupe au groupe des tumeurs et autres lésions en rapport avec l'os. [Kramer, 1992] [1]. Ce groupe comprend d'une part des tumeurs ostéogéniques, d'autre part des lésions osseuses non tumorales. Le fibrome cémentoossifiant est classé parmi les tumeurs ostéogéniques, sous-groupe qu'il forme à lui seul. II est défini comme une néoformation bien limitée, parfois même encapsulée, constituée de tissu fibreux contenant des quantités variables de matériel calcifié ressemblant à de l'os et/ou à du cément. II est caractérisé par une croissance lente et asymptomatique ce qui le rapproche des autres dysplasies fibro-osseuses (dysplasies ostéo-cémentaires et dysplasies fibreuses) parmi lesquelles on a été tenté de le classer.

Waldron [1993] [2] et Zacchariades [1984] [3] ont ainsi proposé d'en faire une lésion tumorale fibroosseuse bénigne se développant aux dépens du ligament desmodontal. Damjanov et Linder [1996] [4] ont suggéré de créer à l'intérieur du vaste groupe fourre-tout des lésions fibroosseuses, un sous-groupe spécifique de lésions fibro-ostéo-cémentaires des maxillaires allant de la dysplasie fibreuse des os à la dysplasie ostéocémentaire péri-apicale floride, incluant le fibrome cémento-ossifiant. Cette tumeur pose donc un problème nosologique qui rend parfois le diagnostic différentiel clinique difficile, en particulier avec la forme monostéotique de la dysplasie fibreuse des os. Le fibrome cémento-ossifiant présente enfin une particularité rare : son aspect histologique peut être comparable à celui de nombreuses formes histopathologiques de la dysplasie fibreuse des os [5]. Le diagnostic doit alors très souvent reposer sur des considérations épidémiologiques, cliniques et radiologiques, ce qui relativise la valeur de référence donnée à l'examen histopathologique et la notion de « diagnostic de certitude » qui lui est souvent inconsciemment associée.

Ces particularités nous ont incités à présenter un cas inhabituel de fibrome cémento-ossifiant du maxillaire chez une femme.

\section{OBSERVATION}

Une femme de 43 ans consulte pour une sensation de gène non douloureuse, localisée dans la partie droite de la cavité buccale. Lors de l'examen clinique, on découvre une tuméfaction vestibulaire maxillaire droite, intéressant la tubérosité et la crête maxillaires édentées, et débordant vers la voûte palatine jusqu'à la ligne médiane (Fig. 1). La tuméfaction sessile est indolore à la palpation, de consistance ferme et homogène. Cette masse non mobile fait corps avec l'os alvéolaire. La muqueuse pala- 


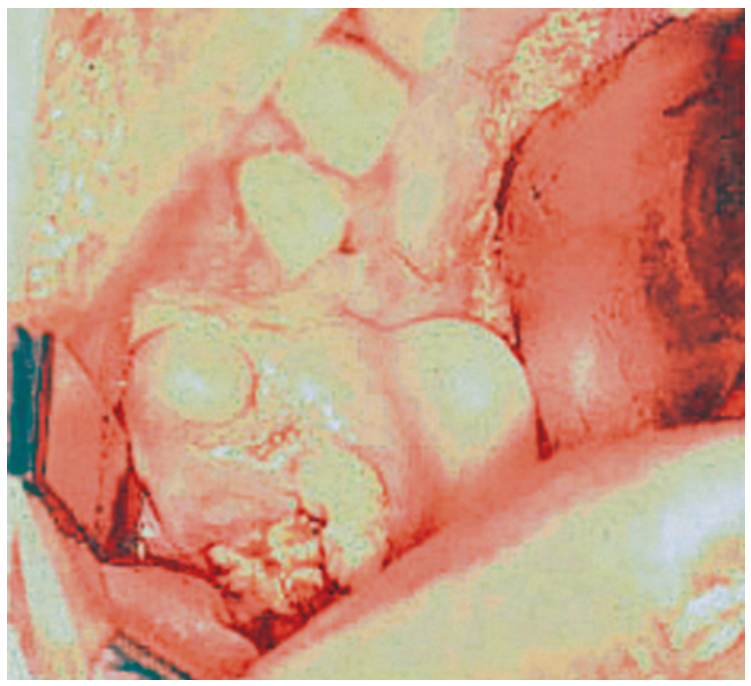

Figure 1 : Aspect clinique de la tuméfaction indurée qui est fixée sur la tubérosité maxillaire droite et s'étend vers la région palatine.

Intraoral view: bony hard fixed swelling from the right maxillary tuberosity to the midpalatine line.

médecine

buccale

chirurgie

buccale

VOL. $10, \mathrm{~N}^{\circ} 1$ 2004

page 23

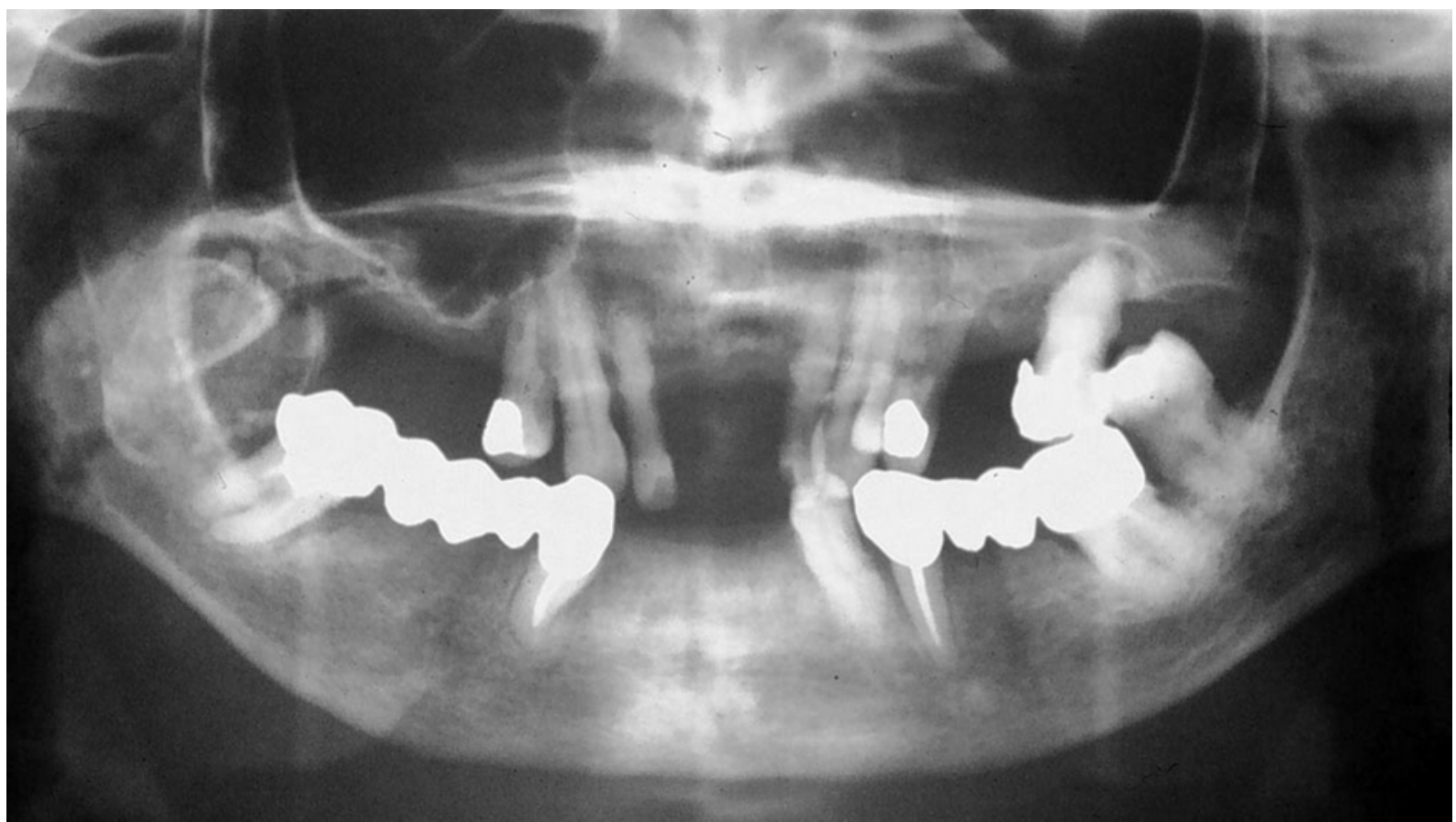

Figure 2 : Orthopantomogramme montrant une image ostéogénique, à pédicule large, implantée dans la zone de la tubérosité maxillaire droite (flèches).

Orthopantomography : A widely pediculated osteogenic image of the right maxillar tuberosity (arrows). 

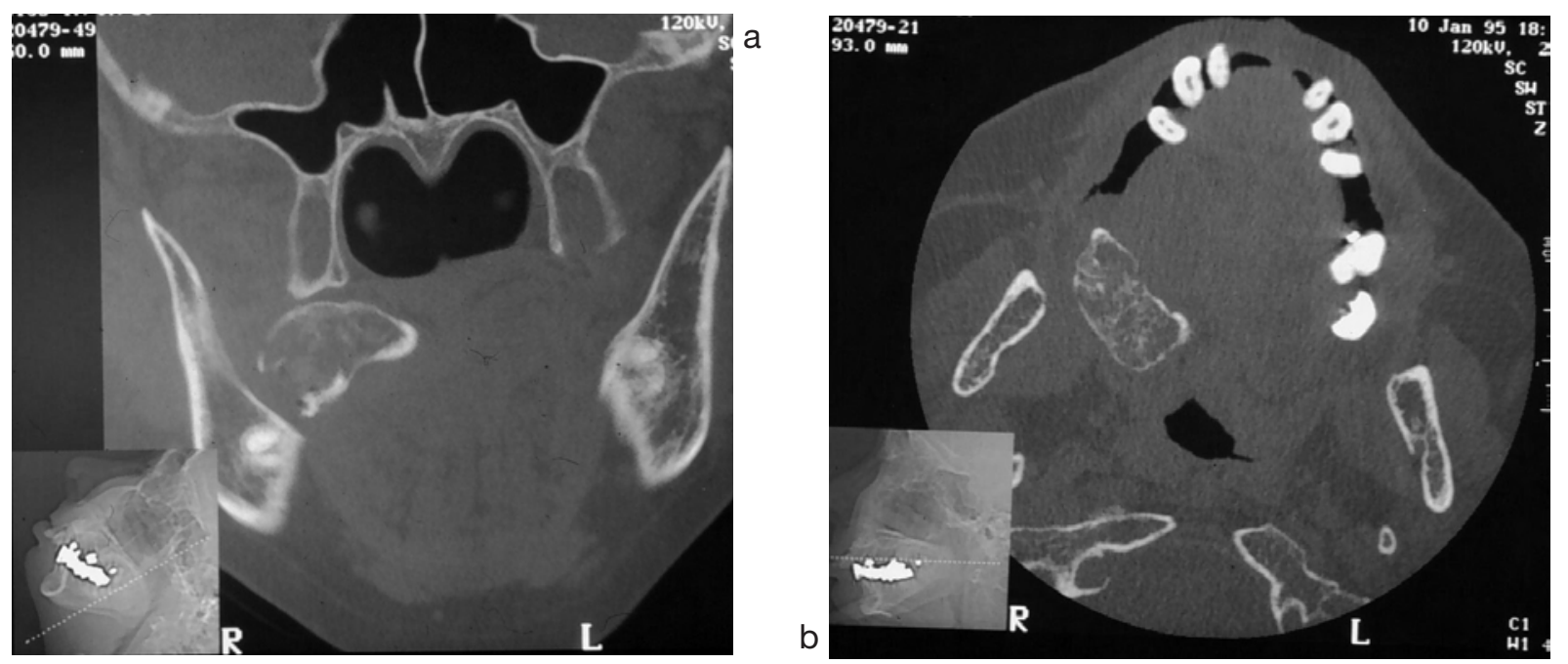

Figures $\mathbf{3 a}$ et $\mathbf{3 b}$ : Coupes TDM coronales et axiales montrant une image ostéogénique, limitée par un liseré de condensation osseuse périphérique, comportant des éléments radio-opaques à disposition radiaire.

Coronal and axial CT slices. The voluminous tumour with bony density is surrounded by a radiopaque margin and contains radiating radiopaque foci.

\section{médecine \\ buccale \\ chirurgie \\ buccale}

VOL. $10, \mathrm{~N}^{\circ} 1$ 2004

page 24

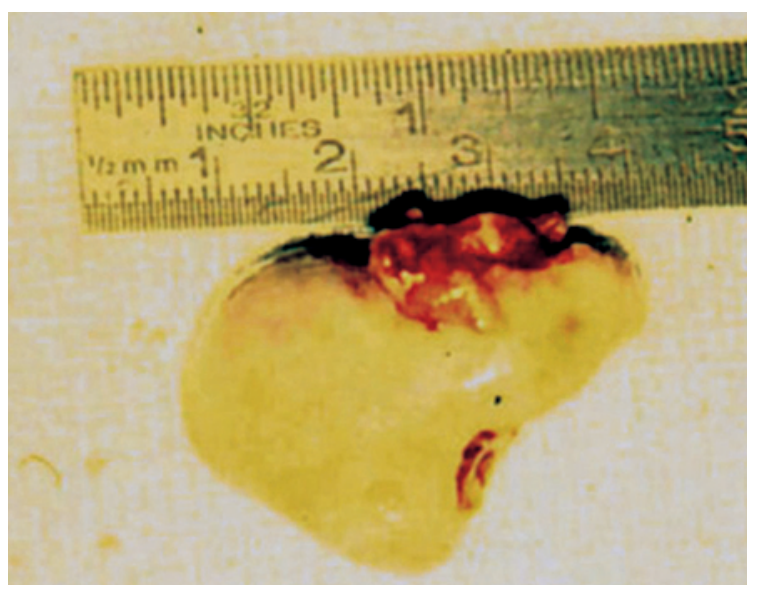

Figure 4 : Pièce opératoire se présentant comme une tumeur osseuse de $3.5 \mathrm{~cm} \times 3 \mathrm{~cm}$ de couleur blanc nacré, lisse.

Macroscopic view of the pearly-white and smooth-surfaced tumour.
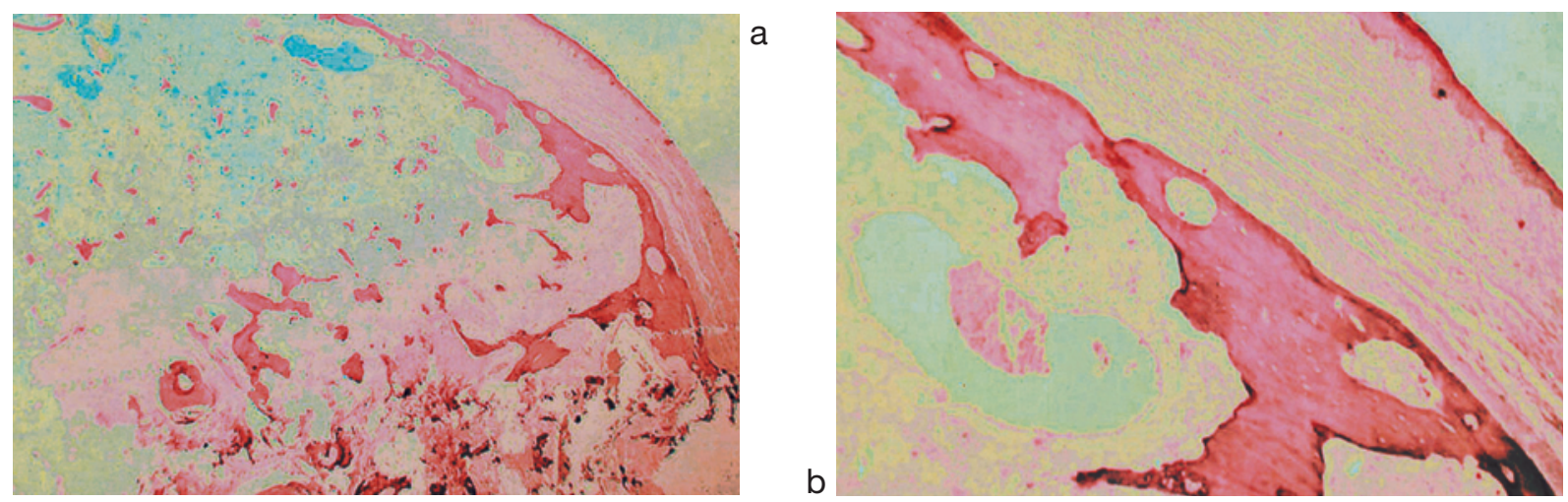

Figures 5 : Coupes histologiques. a : Formations ostéoclastiques dans un stroma mésenchymateux riche en fibroblastes et en travées osseuses lamellaires. b : Travées osseuses avec quelques rares ostéocytes (K) et coque osseuse périphérique $(0)$.

Microscopic views. a : Osteoclastic cells within a mesenchymal stroma with numerous fibroblasts and lamellar osseous trabeculae. $b$ : Osseous trabeculae surrounding a few osteocytes $(O)$; outlying bony capsule(K). 
tine est ulcérée en regard des molaires mandibulaires homolatérales ou prend leur empreinte. Le reste de la muqueuse de recouvrement est normal. L'extraction des dents 15, 16, 17 et 18 est, selon la patiente, bien antérieure à l'apparition de la tuméfaction. L'examen péri-buccal et la palpation des aires lymphatiques sont non contributifs comme le reste de l'anamnèse. L'interrogatoire établit le caractère lentement évolutif de la formation.

L'orthopantomogramme (Fig. 2) montre une formation radio-opaque, exophytique, bordée d'un fin liseré de condensation, qui semble appendue à la crête alvéolaire maxillaire.

Au sein de cette formation, on retrouve des plages d'ostéolyses associées à des travées concentriques de densité osseuse à peine discernable. La partie postérieure de cette néo-formation est adjacente à un reste radiculaire de la 18. L'examen tomodensitométrique (Fig. 3a et 3b) confirme le caractère bien délimité de la lésion qui est entourée d'une corticale osseuse légèrement dense à la périphérie. On retrouve des inclusions sous forme de trabécules denses, jouxtant des lacunes osseuses. Cette formation semble se développer aux dépens du seul maxillaire, sans envahissement des régions adjacentes. Le diagnostic clinique et radiologique de fibrome cémento-ossifiant est alors proposé.

Le traitement chirurgical, réalisé sous anesthésie générale, consiste en une énucléation totale de la tumeur. La voie d'abord vestibulaire laisse apparaître une tumeur dont le clivage est relativement aisé. Les suites opératoires sont simples et la patiente, suivie jusqu'à ce jour, ne présente aucun signe de récidive.

Sur le plan macroscopique (Fig. 4), la pièce opératoire mesure $3,5 \mathrm{~cm}$ de diamètre. Elle est ovalaire, d'aspect éburné et de consistance dure, de couleur et de nature évoquant l'os. L'examen microscopique (Fig. $5 a$ et $5 b$ ) révèle une prolifération fibroblastique périphérique, enserrant des structures ostéoblastiques avec ossification imparfaite, s'organisant par endroits en trabécules réguliers dans un tissu conjonctif fibreux. La muqueuse susjacente est amincie, discrètement inflammatoire et normalement kératinisée. Des travées osseuses sont retrouvées au sein d'un mésenchyme riche en fibroblastes et peu vascularisé. Ces travées sont agencées en réseau, bordées par des rangées d'os- téoblastes et de quelques rares ostéoclastes. On retrouve des ostéocytes, enchâssés dans des logettes, parsemant la pièce opératoire. Le diagnostic histopathologique de fibrome cémento-ossifiant est confirmé.

\section{COMMENTAIRES}

Menzel (1872) fut le premier à décrire le fibrome ossifiant. Le terme fut utilisé par Montgomery en 1927 pour décrire une lésion bien délimitée survenant au sein des structures osseuses du massif facial et produisant des néoformations osseuses et fibreuses. Par la suite, la terminologie a considérablement varié: ostéome fibreux, fibrome sclérosant, ostéite hypertrophique ou fibreuse localisée, ostéodystrophie fibreuse localisée et chéloïde osseuse sont autant de dénominations qui ont été utilisées pour cette lésion [6,7]. À partir de 1946, Schlumberger propose une classification unitaire des lésions fibroosseuses qui inclut le fibrome ossifiant comme une variante de la dysplasie fibreuse des os [8]. Cahn (1951), en décrivant des calcifications ovoïdes d'aspect cémentiforme dans du tissu fibreux, distingue deux nouvelles entités, le fibrome ossifiant et le fibrome cémento-ossifiant, qu'il différencie clairement de la dysplasie fibreuse des os [6].Cette approche, corroborée par l'ensemble des travaux histologiques et histochimiques qui ont suivi, aboutit en 1992 dans la classification de l'OMS à l'individualisation du fibrome cémentifiant et du fibrome ossifiant. Ils représenteraient respectivement une tumeur en rapport avec l'appareil odontogénique et une néoformation sans relation avec l'appareil odontogénique.

En réalité, cette tentative d'individualisation a fait long feu. Dès 1992, dans leurs commentaires de la classification, Kramer et coll. soulignent qu'il est souvent difficile de distinguer le cément de l'os, deux tissus qui, de plus, ont la même origine histogénétique [1]. Eversole et coll. (1985) [9] puis Damjanof et Linder (1996) [4] jugent cette séparation arbitraire et inutile et ils proposent de rassembler les deux entités sous la dénomination de fibrome ossifiant.

Cette question n'est toujours pas tranchée mais, depuis 1992, la plupart des auteurs utilise le terme médecine

buccale

chirurgie

buccale

VOL. $10, \mathrm{~N}^{\circ} 1$ 2004

page 25 
médecine

buccale

chirurgie

buccale

VOL. $10, \mathrm{~N}^{\circ} 1$ 2004

page 26 de fibrome cémento-ossifiant (Sapp et coll. 1997 [10], Wood et Goaz 1997 [11], Cawson et Odell 1998 [12], Cawson et Binnie 1999 [5], Reichart 1999 [13]) réservant celui de fibrome ossifiant aux rares formes extragnathiques - fibrome ossifiant juvénile et fibrome ossifiant périphérique - qui en dépit de son homonymie est une entité histologiquement radicalement différente.

Le fibrome cémento-ossifiant est une tumeur bénigne des os ayant une ossification membraneuse. II touche donc exclusivement les os du squelette maxillo-facial [14,15]. II apparaît le plus souvent monostotique mais il existe des formes multiples à partir de cas sporadiques ou plus rarement familiaux [16,17]. II survient généralement entre la deuxième et la quatrième décade, avec un ratio homme/femme de $1 / 5$. Sa localisation est mandibulaire dans $75 \%$ des cas, intéressant la région prémolo-molaire $[8,18,19,20]$. II se révèle par une tuméfaction osseuse de croissance lente, progressive, indolore et provoque parfois le refoulement des organes dentaires sans entraîner de rhizalyse. La vitalité des dents adjacentes est préservée. Il refoule également les tissus avoisinants sans les détruire. Sa consistance est plus ou moins ferme selon son degré de minéralisation. Le fibrome cémento-ossifiant est recouvert d'une muqueuse normale. On ne retrouve pas de signes généraux ni d'adénopathies associés [21].

Le tableau clinique est très proche de celui de la dysplasie fibreuse dans sa forme monostotique, ce qui explique les difficultés diagnostiques au stade initial. La transformation maligne est extrêmement rare [8].

Classiquement, l'aspect radiologique est initialement caractérisé par une image ostéolytique, bien délimitée, se distinguant aisément de l'os environnant [19,22].

À la mandibule, il s'agit d'une lacune refoulant le canal mandibulaire qui reste intact, et amincissant les corticales vestibulaire et linguale [21].Le fibrome cémento-ossifiant peut être découvert à un stade précoce, sous l'aspect d'une image bien circonscrite, essentiellement radioclaire, prenant parfois même un aspect pseudo-kystique. Au cours de l'évolution, de fines opacités vont se développer au centre de la lésion ; elles ont une densité plus faible que celle de l'os environnant, mais elles peuvent être découvertes précocement par l'examen tomodensitométrique [21,23]. À un stade de maturation plus avancé, l'image est composée d'opacités irrégulières formant des travées osseuses concentriques, circonscrites par une ostéocondensation périphérique, souvent comparée à une coquille d'œuf [8,23]. Les travées osseuses ont une disposition plutôt radiaire par rapport au centre de l'image mais, au terme de l'évolution, on aboutit à une opacité presque complète de la lésion. Au maxillaire, la lésion peut être découverte plus tardivement si son développement se fait de façon asymptomatique dans le sinus maxillaire et/ou dans les fosses nasales. L'exploration tomodensitométrique en fenêtre osseuse permet de faire le bilan d'extension de la tumeur et d'évaluer le refoulement des tissus environnants. On retrouve, volontiers dans les localisations maxillaires, un aspect uni- ou multiloculaire, bien délimité par une coque osseuse d'épaisseur variable. Des cloisons osseuses forment de véritables logettes au contenu hypodense. Les structures avoisinantes sont refoulées, sans destruction. À ce stade d'évolution, le fibrome cémento-ossifiant présente des similitudes radiologiques avec la dysplasie fibreuse, ce qui complique son diagnostic [24]. Quoiqu'il en soit, l'aspect bien délimité d'une image ostéolytique pure ou en demi-teinte est évocateur de la lésion, et doit être un élément évoqué lors du diagnostic différentiel avec la dysplasie fibreuse. L'IRM complète les informations obtenues par la TDM. La coque osseuse périphérique apparaît hypo-intense en T2, et se rehausse après injections de produit paramagnétique. Ce rehaussement permet de différencier la formation tumorale et les tissus mous environnants (muqueuse sinusienne...) [24].

Au cours de son évolution, l'aspect macroscopique de la lésion passe progressivement d' une masse de consistance molle, d'allure kystique, à une formation osseuse, dure, de couleur blanc nacré [22].

Les descriptions histopathologiques du fibrome cémento-ossifiant colligées $[22,25,26]$ sont compatibles avec celle de cette observation. Sur le 
plan histologique, on observe une double composante : osseuse et mésenchymateuse. Des travées osseuses, élaborées au sein d'un mésenchyme presque toujours cellulaire, sont agencées en réseau [22,25,27]. Le collagène est disposé en faisceaux parallèles, implantés sur les travées osseuses. L'examen en lumière polarisée permet de retrouver cette trame régulière au sein du tissu osseux. On observe de petits foyers de cellules géantes [19, 28]. Au cours de la maturation, les îlots d'ossification croissent en nombre et en volume et ils finissent par confluer. Ceci explique l'évolution constante de l'aspect radiologique vers une radio-opacité toujours plus marquée [6].

L'étude histo-enzymologique du fibrome cémentoossifiant révèle une activité élevée des enzymes oxydatives du cycle de Krebs. Parallèlement, il existe une activité croissante de la phosphatase alcaline sérique et de l'ATPase dans les cellules fibroblastiques du mésenchyme qui ont de nombreuses expansions cytoplasmiques. La présence des réactions ATPasiques et des phosphatases alcalines démontre une activité fonctionnelle de la lésion qui édifie une trame osseuse selon un processus d'ossification normal [6]. La minéralisation, d'intensité variable, est par endroits peu abondante, périfibrillaire, prenant un aspect uniforme basophile, acellulaire et ovoïde. Parfois, plus massive, elle se dispose en larges plaques calciques, lamellaires, parsemées de lacunes englobant des ostéocytes préexistants [22]. L'histopathologie du fibrome cémento-ossifiant oblige à revenir sur le débat terminologique abordé plus haut. Pour Cahn, les lésions dont les calcifications sont formées par de l'os lamellaire, représentent les fibromes ossifiants, alors que celles dont les calcifications sont amorphes, basophiles, et donc considérées comme constituer par du cément, correspondent à des fibromes cémentifiants [18,19,29]. Les nombreuses lésions qui contiennent les deux types de calcification sont des fibromes cémento-ossifiants [16]. Sciubba et Younai ont montré que la présence ou non de cément au sein de la lésion n'a aucune influence sur l'aspect radiologique, ni sur la clinique et moins encore sur le taux de récidive [22].

L'os et le cément sont deux entités microscopiquement distinctes, mais l'origine des cémento- blastes et des ostéoblastes est commune. II s'agit du recrutement de cellules issues de la membrane desmodontale - elle-même d'origine membraneuse - qui produit également des fibroblastes [22]. Cette hypothèse a été confirmée par les travaux de Cho et coll. (1988), qui ont utilisé les EGF (Epithelial Growth Factors) comme marqueurs exprimés par les préostéoblastes, les précémentoblastes et les préostéocytes, en plus des fibroblastes issus de la membrane desmodontale [22].Dans une étude portant sur un cas familial de fibromes cémento-ossifiants multiples chez une jeune fille, Yih et coll. (1989) ont également décrit des calcifications cémentiformes [17]. Enfin, des calcifications identiques ont été trouvées dans des lésions fibro-osseuses intéressant la base du crâne et d'autres os du squelette, à distance de tout tissu odontogénique [17,18].

Selon Mesquita et coll. (1998), une influence hormonale expliquerait l'apparition du fibrome cémento-ossifiant chez la femme adulte alors qu'elle est beaucoup plus rare avant 10 ans et exceptionnelle après 40 ans. Les auteurs ont mis en évidence l'activité proliférative de cette tumeur en utilisant la méthode d'analyse morphométrique et quantitative de l'Ag NOR (Nucleolar Organizer Region). Le NOR est une boucle d'ADN transcrite en ARN ribosomal et en protéine. Cette boucle est associée à des protéines spécifiques qui peuvent être visualisées par une fixation à l'argent. Cette étude montre que le fibrome cémento-ossifiant présente les caractéristiques des lésions bénignes avec néanmoins une activité proliférative élevée [30]. Etant donné la nature des cellules qui le composent et l'activité métabolique de celles-ci, le fibrome cémento-ossifiant représente donc un authentique processus tumoral.

Le diagnostic différentiel pose souvent un problème complexe. L'absence des signes cliniques évocateurs permet d'éliminer d'emblée toute pathologie inflammatoire ou traumatique aiguë. L'évolution lente et progressive, indolore et sans trouble neurologique associé, plaide en faveur d'un processus néoplasique bénin. L'évolution constante de l'image radiologique incite à élaborer un diagnostic différentiel en fonction des différents stades de maturation du fibrome médecine

buccale chirurgie buccale

VOL. $10, \mathrm{~N}^{\circ} 1$ 2004

page 27 
médecine

buccale

chirurgie

buccale

VOL. $10, \mathrm{~N}^{\circ} 1$ 2004

page 28 cémento-ossifiant. Sa localisation au sein des maxillaires et son rapport étroit avec les dents doivent faire évoquer successivement une origine non odontogénique, puis odontogénique [21]. Parmi les lésions radiotransparentes non odontogéniques, on distingue :

- Le fibrome ossifiant qui représente une forme agressive à développement extragnathique et qui, à partir d'une localisation généralement maxillaire, aboutit à l'envahissement des sinus paranasaux, des cavités orbitaires, des os frontaux et des structures de la base du crâne. Cette évolution sévère, infiltrante, peut entraîner une asymétrie faciale, une exophtalmie, une obstruction des fosses nasales [8, 23,31].

- Le fibrome ossifiant juvénile est une tumeur de l'enfant, douloureuse et à croissance rapide. Elle présente un potentiel destructeur important et une rapidité d'évolution bien supérieure à celle du fibrome cémento-ossifiant [21]. Le taux de récidive après traitement est élevé [32]. L'aspect histologique diffère du fibrome ossifiant par une plus grande richesse cellulaire, une disposition tourbillonnante des cellules fusiformes, et des formations osseuses de type trabéculaire, plutôt que lamellaire [32].

En dehors de ces formes cliniques particulières, d'autres lésions peuvent être envisagées : le kyste anévrysmal d'évolution rapide et douloureuse ; le kyste osseux solitaire et le granulome à cellules géantes dont l'âge de survenue est plus précoce [21]. Les lésions vasculaires seront écartées en raison de leur croissance rapide, de leur survenue précoce (hémangiome) et des bruits vasculaires perceptibles (malformations artérioveineuses) [21]. Parmi les lésions odontogéniques, on doit évoquer la tumeur odontogénique calcifiée de Pindborg, la tumeur odontogène adénomatoïde et le kératokyste odontogénique. Le plus souvent, ces lésions ne poseront pas de véritable problème de diagnostic différentiel sur la base de leur localisation préférentielle ou de leur évolution rapidement agressive.

Ce sont surtout les images radiologiques mixtes qui doivent faire évoquer les tumeurs fibroosseuses des maxillaires (Tab. 1) et tout particulièrement les dysplasies ostéo-cémentaires péri-apicales et la dysplasie fibreuse des os. Comme la dysplasie ostéo-cémentaire péri-apicale. Le fibrome cémento-ossifiant peut survenir dans la région apicale de dents vivantes. II est rencontré le plus souvent à la mandibule et il passe par des stades de maturation voisins de ceux de la dysplasie ostéo-cémentaire péri-apicale. Mais les deux entités s'opposent par le fait que le fibrome cémento-ossifiant se développe

Tableau 1 : Classification des lésions fibro-osseuses, d’après Wood et Goaz, 1997 [11].

I. Dysplasie fibreuse

- dysplasie fibreuse monostotique

- dysplasie polyostotique

II. Dysplasies réactionnelles de la région alvéolo-dentaire

Elles sont présumées dériver du desmodonte. On les sépare volontiers en 3 entités en fonction de leur aspect radiologique bien qu'elles relèvent d'un processus histopathologique identique.

- dysplasie ostéo-cémentaire périapicale

- dysplasie ostéo-cémentaire focale

- dysplasie ostéo-cémentaire floride

III. Tumeurs fibro-osseuses

- fibrome cémento-ossifiant

- fibrome ossifiant (agressif, extragnathique)

- fibrome ossifiant juvénile 
chez des sujets plus jeunes, que la dysplasie ostéo-cémentaire péri-apicale prédomine dans la région incisivo-canine, que les lésions de la dysplasie ostéo-cémentaire péri-apicale sont de petite taille, comparées à celles du fibrome cémento-ossifiant.

Le diagnostic différentiel essentiel se fait avec la dysplasie fibreuse ; il repose sur des critères radiologiques, histologiques, voire chirurgicaux. Contrairement au fibrome cémento-ossifiant, la dysplasie fibreuse présente une image radiologique mal limitée, avec un aspect en verre dépoli. Pour certains auteurs $[23,26]$, la radiographie conventionnelle suffit à différencier les deux entités ; le diagnostic repose sur des signes radiologiques et histologiques compatibles avec une lésion fibroosseuse. D'un point de vue anatomo-pathologique, ces deux lésions sont en effet difficilement différenciables à un stade précoce. Néanmoins, dans la dysplasie fibreuse, l'ossification est anormale, irrégulière, sans ostéoblastes périphériques. On retrouve des cellules fibroblastiques dysplasiques, associée à une trame collagénique irrégulière, de nombreuses cellules géantes et des zones hémorragiques $[6,33]$. Ces différents caractères, apparaissant au stade évolué de la dysplasie, permettent finalement de la différencier du fibrome cémentoossifiant. Enfin, sur le plan clinique, le clivage aisé du fibrome cémento-ossifiant contraste avec l'absence de limites nettes entre la dysplasie et l'os sain : cet élément a une incontestable valeur diagnostique. D'ailleurs, après énucléation complète, la récidive du fibrome cémento-ossifiant est rare alors qu'elle atteint un taux de $25 \%$ pour la dysplasie fibreuse. Ce résultat est probablement lié à une élimination incomplète de la lésion qui justifie, pour certains, la résection en bloc dans les formes monostéotiques [18,23].

Le traitement est chirurgical, parfois précédé d'une cytoponction notamment dans les lésions radio-claires. L'énucléation, complétée par un curetage de la cavité, constitue le traitement de première intention du fibrome cémento-ossifiant avec une surveillance clinique et radiologique [27].
Le taux de récidive est très faible ${ }^{[3,22]}$ bien qu'une série ait curieusement présenté un taux de récidive de $28 \%$ [27].

Le fibrome cémento-ossifiant est une tumeur bénigne des os de membrane qui pose à la fois un problème pour sa nosologie et son diagnostic différentiel.

La littérature et la clinique poussent à ne plus séparer cette entité en deux lésions, ossifiante et cémentifiante : d'abord parce que la clinique est indépendante de la nature du tissu calcifié prédominant, ensuite parce que l'histogénèse et l'aspect histopathologique de ces deux tissus, os et cément, les rend difficilement discernables. II convient donc de qualifier cette tumeur bénigne de fibrome cémento-ossifiant et de réserver le terme de fibrome ossifiant aux formes juvéniles, agressives et extragnathiques. Le fibrome cémento-ossifiant est rattaché aux lésions à point de départ desmodontal.

Le regroupement de cette entité avec les dysplasies fibro-osseuse qui comportent les dysplasies fibreuses et les dysplasies ostéo-cémentaires, paraît logique compte tenu à la fois de la grande similitude histopathologique initiale de ces lésions et de leur comportement clinique globalement peu agressif. Sur le plan histopathologique, au stade initial, le matériel fibreux du fibrome cémento-ossifiant a une organisation lamellaire, celui de la dysplasie fibreuse est composé de trousseaux de fibres torsadés. La distinction avec la dysplasie ostéo-cémentaire péri-apicale, qui est de plus petite taille, repose sur la présence de limites nettes dans le fibrome cémento-ossifiant alors que celles de la dysplasie ostéo-cémentaire péri-apicale sont peu marquées et cette dernière peut apparaître sans véritable discontinuité avec l'os environnant.

D'une manière plus générale, le fibrome cémentoossifiant soulève le problème d'une révision profonde de la classification OMS en vue de regrouper, selon des critères étiopathogéniques, l'ensemble des lésions dites à point de départ desmodontal. médecine

buccale

chirurgie

buccale

VOL. $10, \mathrm{~N}^{\circ} 1$ 2004

page 29 


\section{RÉFÉRENCES}

1 - Kramer IRH, Pindborg JJ, Shear M. Histologic typing of odontogenic tumours (pp 27-28). Springer-Verlag, Berlin, 1992.

2 - WALDRON CA. Fibro-osseous lesions of the jaws. J Oral Maxillofacial Surg 1993; 51: 828-35.

3 - Zacchariades N, VairaKtaris E, PAPANICOLAOU S. Ossifying fibroma of the jaws: review of the literature and report of 16 cases. Int J Oral Surg 1984; 13: 1-6.

4 - DAMJANOV I, LINDER J. Anderson's pathology (pp 16061607). Mosby, St Louis, 1996.

5 - Cawson RA, Binnie WH, Speight PM, BarRett A.W, WRIGHT JM. Luca's pathology of tumours of the oral tissues (pp 156). Churchill Livingstone, Londres, 1999.

6 - Jammet P, CAndon B, Montes de OCA, Souyris F. Quelques cas de fibromes ossifiants et de dysplasies fibreuses cranio-maxillo-faciaux. Rev Stomatol Chir Maxillofac 1987; 88: 10-4.

7 - Piette E, Reychler H. Traité de pathologies buccale et maxillo-faciale (pp 1384-1385). De Boeck-Wesmael, Bruxelles, 1991.

médecine buccale chirurgie buccale

VOL. $10, \mathrm{~N}^{\circ} 1$ 2004

page 30
8 - Commings DJ, Tolley NS, Milford CA. Fibrous dysplasia and ossifying fibroma of the paranasal sinuses. J Laryngol Otol 1998; 112: 964-8.

9 - Eversole LR, LeIDER AS, Nelson K. Ossifying fibroma: a clinicopathologic study of sixty four cases. Oral surg Oral Med Oral Path 1985; 60: 505-17.

10 - SAPP JP, EVERSOLE LR, WYSOCKI GP. Contemporary oral and maxillofacial pathology (pp 109-110). Mosby, St Louis, 1997.

11 - Wood NK, GoAz PW. Differential diagnosis of oral and maxillofacial lesions (pp 328-329). Mosby, St Louis, 1997.

12 - CAWSON RA, OdELL EW. Essentials of oral pathology and oral medecine (pp 133-135). Churchill Livingstone, Londres, 1998.

13 - REICHART PA, PHILIPSEN HP. Oralpathologie (pp 192). Georg Thieme Verlag, Stuttgart, 1999.

14 - Guilbert F, Chomette G, Le Charpentier Y, Auriol A. Les tumeurs bénignes et pseudo-tumeurs des maxillaires. Rev Stomatol Chir Maxillofac 1993; 94: 222-9.

15 - Buchet C, Baralle MM, Gosset P, Lecomte-Houcke M, DonazZAN M. Fibrome ossifiant maxillaire : à propos de trois cas. Rev Stomatol Chir Maxillofac 1994; 95: 95100.

16 - Hauser M, Freije S, Payne R, Timen S. Bilateral ossifying fibroma of the maxillary sinus. Oral Surg Oral Med Oral Pathol 1989 ; 68 : 759-63.

17 - YIH W, PEDRESON G, BARTLEY M. Multiple familial ossifying fibromas: relationship to other osseous lesions of the jaws. Oral Surg Oral Med Oral Pathol 1989; 68: 754-8.
18 - WALDRON C. Fibro-osseous lesions of the jaws. J Oral Maxillofac Surg 1985; 43: 249-62.

19 - Hall E, NAYLOR G, Mohr R, Warnock G. Early agressive cemento-ossifying fibroma: a diagnostic and treatment dilemma. Oral Surg Oral Med Oral Pathol 1987; 63: 132-6.

20 - Rosenberg A, Mokhtari H, Slooweg PJ. The natural course of an ossifying fibroma. J Oral Maxillofac Surg 1999; 28: 454-6.

21 - Halkias L, Larsen P, Allen C, SteinBerg M. Rapidly growing, expansile mass of the mandible in a 6-years-old boy. J Oral Maxillofac Surg 1998; 56: 866-71.

22 - ScIUBBA J, YounAl F. Ossifying fibroma of the mandible and maxilla: review of 18 cases. J Oral Pathol Med 1989; 18: 315-21.

23 - Burns J, LezZoni J, Reibel J. Pathologic quiz case 2. Arch Otolaryngol Head Neck Surg 1996; 122: 681-3.

24 - MARTIN-DUVERNEUIL N, CHIRAS J. Imagerie maxillo-faciale (pp 100-102) . Médecine-Sciences - Flammarion, Paris, 1997.

25 - KadiRI F, LaraquI NZ, TouhanI M, Benghalem A, MOKRIM B, CHEKKOURY-IDRISI A, BenCHAKROUN Y. Les fibromes ossifiants des maxillaires. Rev Laryngol 1993; 114: 349-53.

26 - Sakuma T, KaWASAKI T, Watanabe K. Concurrent cementifying and ossifying fibromas of the mandible: report of a case. J Oral Maxillofac Surg 1998; 56: 778-82.

27 - EverRSOLE LR, LeIDER AS, Nelson K. Ossifying fibroma: a clinicopathologic study of sixty-four cases. Oral Surg Oral Med Oral Pathol 1985; 60: 505-11.

28 - William K, Manghan C, SpeIGHt M. Juvenile ossifying fibroma. An analysis of eight cases and comparison with other fibro-osseous lesions. J Oral Pathol Med 2000; 29: 13-8.

29 - MAYO K, ScotT R. Persistent cemento-ossifying fibroma of the mandible: report of a case and review of literature. J Oral Maxillofac Surg 1988; 46: 58-63.

30 - Mesquita R, Orinin S, Sousa M, Soraes de Araujo N. Proliferative activity in peripheral ossifying fibroma and ossifying fibroma. J Oral Pathol Med 1998; 27: 64-7.

31 - WenIG BM, VINH TN, SMIRNIOTOPOULOS JG, FOWLER CB, Houston GD, HefFNER DK. Aggressive psammomatoid ossifying fibroma of the sinonasal region. Cancer 1995; 76:1155-65.

32 - HARNet JC, FeKI A, KHAN JL. Fibrome ossifiant juvenile: à propos d'un cas. Med Buc Chir Bucc 1997; 3: 27-32.

33 - Chomette G, Auriol M, Boisnic S, Guilbert F, VAILANT JM. Dysplasie fibreuse et fibrome ossifiant : critères morphologiques. Rev Stomatol Chir Maxillofac 1987; 88: 1-7. 